\title{
Research of Power Grid Fault Diagnosis and Intelligent Analysis Method Based on Multi-Source Information
}

\author{
Qingquan $\mathrm{Wu}^{1, \mathrm{a}^{*}}$, Le Huang ${ }^{1, \mathrm{~b}}$ and Houbing Deng ${ }^{1, \mathrm{c}}$ \\ ${ }^{1}$ No116, Tianhe Road, Tianhe District, Guangzhou, Guangdong, PR China, 510000 \\ atsingquen@hotmail.com, bhuangle@ehv.csg.cn, denghoubing@ehv.csg.cn
}

Keywords: Integration of scheduling and control; Energy management system; Fault diagnosis; Intelligent analysis; Multi-source information

\begin{abstract}
With the integration of scheduling and control promote, the warn processing function of existing power dispatching automation system such as energy management system need improved. In order to facilitate the supervisor of Power Dispatch and Centralized Control Center to understand the operation state of the power grid accurately, the warn processing function distributed in each system should be processed synthetically. This paper presents a noval method for Power system fault diagnosis and intelligent analysis based on fusion of multi-source information; it can determine the location of the fault quickly then make reference decisions. It can not only improve the efficiency of supervisor but also avoid accident and abnormal further expansion.
\end{abstract}

\section{Introduction}

Power Dispatch and Centralized Control Center usually configured with Supervisory Control and Data Acquisition (SCADA) system, Power Fault Analyzing and Processing System (PFAP), Fault Recorder systems and so on, each system is independent, warn information distributed in all of them, lack of effective integration. When fault occurs, the supervisor needs to make a association analysis according to lager number of data [1], it's inefficiency that analysis depend on human experience. The fault diagnosis method based on Artificial Intelligence algorithm, such as Fuzzy Logic and Genetic Algorithm, plays an important role in improving the intelligent level of warn processing [2] [3] [4], but it can't adopted to the development requirements of integration of scheduling and control, the algorithm above is based on single-source, the accuracy of fault analysis affected by the quality of basic data, it diagnose the simple fault quickly, but in case of complex fault such as Relay protection or breaker action reject needed further study. This paper propose a diagnosis and intelligent analysis method Based On mutil-Source Information, it combines steady state, dynamic state and transient state of power grid to achieve the function.

\section{System Architecture}

\section{Network Architecture.}

1. Substation

Substation Device includes Measuring and Control Device, Relay Protection Device, Phase Measurement Unit (PMU), Fault Recorder etc. They used for monitor, measure and control the primary equipment of power grid. Remote Terminal Unit (RTU) used for information collection and transmission between substation and dispatching center.

2. Dispatching Center

Secondary Security Partition is divided into three partitions:

Partition I, Real-time Control Area, Front End System (FES), SCADA, Advanced application modules such as State Estimation install on Power Application Software (PAS) .

Partition II, Uncontrolled Area, include PFAP, Fault Recorder, Wide Area Measurement System (WAMS). The information of Fault Recorder uploads to PFAP.

Partition III, Production Management Area, it mainly include WEB, in charge for publishing information to LAN users, data originates from Partition I or II, the isolator limits the flow of data from Zone I or Zone II only. 


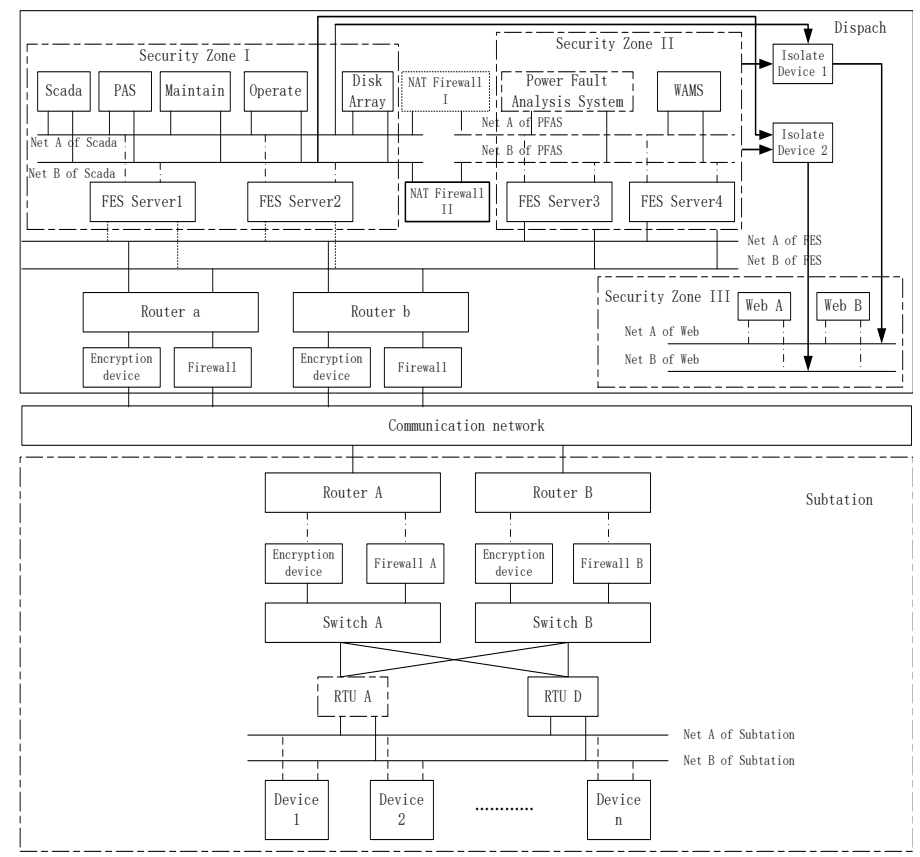

Figure 1. Network Architecture

\section{Network Architecture.}

There are four types of alarm information sources:

1. Steady State Information, including Sequence Of Event (SOE), Total Accident, Breaker Position Altering, Protection Action; good performance in real-time, wide distribution, the results only include failure time, equipment reclosing situation.

2. Dynamic Data, Less distribution, the analysis results can be obtained fault phase based on the Steady-State information.

3. Transient data, the analysis results can be obtained Fault location, Short circuit current and other information based on the Steady-State information.

4. Message of Relay Protection Device, used to judge the fault property.

In summary, through integration of redundant multi-source information, it can solve the problem of false alarm caused by single-source information and improve the performance of fault diagnosis effectively [5] [6] [7] [8].

\section{Fault Diagnosis Logic}

\section{Information Correctness Verification.}

1. Steady State information correctness verification. By using inherent relationship of Remote singal such as breaker position change, total accident, relay protection action, establish identification rules to realize correctness verification: When equipment maintenance, total accident Act, Relay protection didn't Act, we can realize that total accident signal missed according to the rules [9].

2. Transient State information correctness verification. Get the fault time, fault equipment According to the warning information, integrate other warning information of fault equipment within same time range, including Steady State information and Fault briefing, and verify the correctness according to the rules.

\section{Fault Diagnosis Base on Steady State Information.}

The main basis includes Remote Signal, such as breaker position change, total accident, etc. Remote Measurement, such as electric current, voltage and power. The following fault diagnosis logic, requirements: the difference of signal time in the same substation less than 20 seconds, different substations no more than 60 seconds.

1. Fault diagnosis logic for power transmission line 


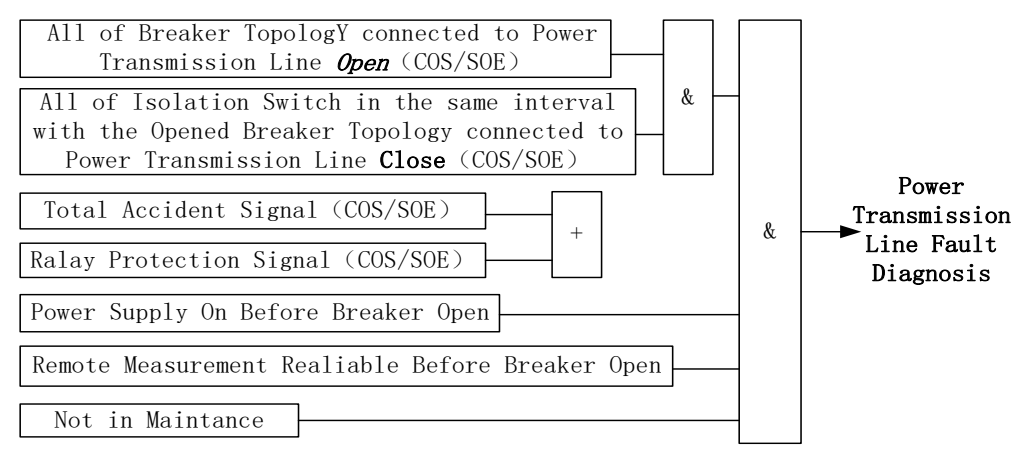

Figure 2. Fault diagnosis logic for power transmission line

$3 / 2$ connection mode, if one of the breaker in the maintenance, there is only one breaker trip when fault occurs. Remote measurement credible means two minutes before the fault occur, average value of power is greater than zero floating.

3. Fault diagnosis logic for buses

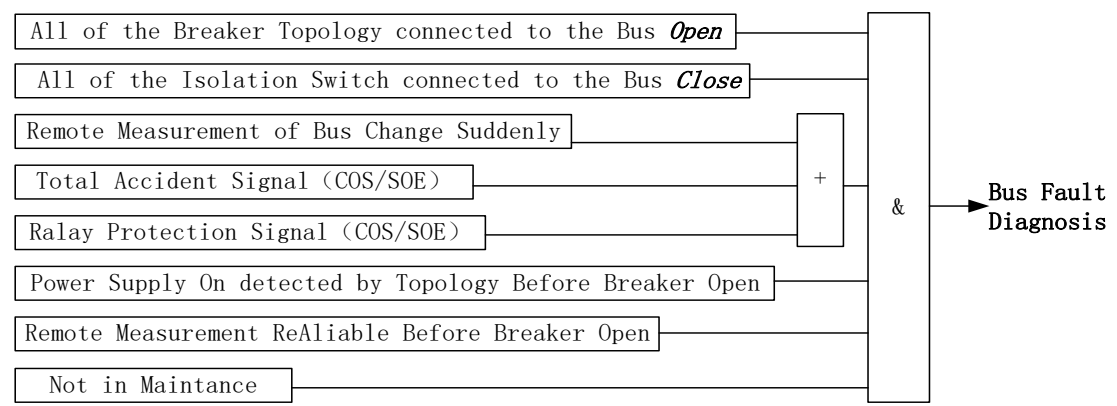

Figure 3. Fault diagnosis logic for buses

If there is a breaker in maintenance, the number of breakers in maintenance and the number of breakers trip is equal to the number of breakers topology connected to the bus. Remote measurement correct means two minutes before the fault occur; the average voltage of bus is more than $80 \%$ of the rated value.

4. Fault diagnosis logic for transformer

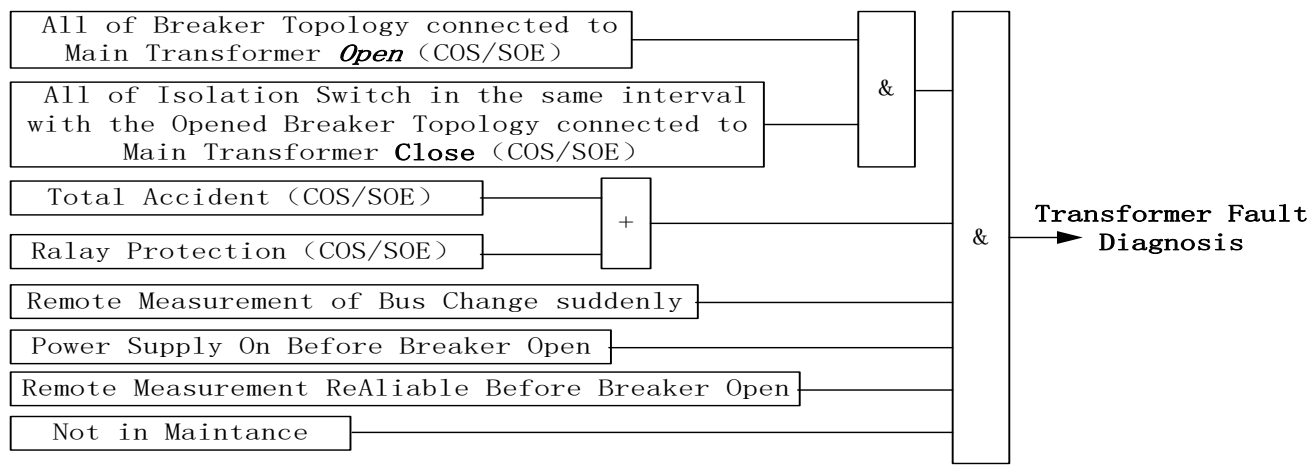

Figure 4. Fault diagnosis logic for transformer

Remote Measurement change suddenly, the power value down exceeds 5\% of the power rated value, the mutation moment and the fault occur moment is not more than 1 minutes.

Fault Diagnosis base on Dynamic State Information. The data source is mainly from the short circuit fault identified by on-line disturbance identification, after the short circuit fault information is received, Judge whether the remote signal that break trip in 5 minutes, if meet the above conditions, then send out equipment failure alarm, then the fault alarm will be sent out if the condition is satisfied. 


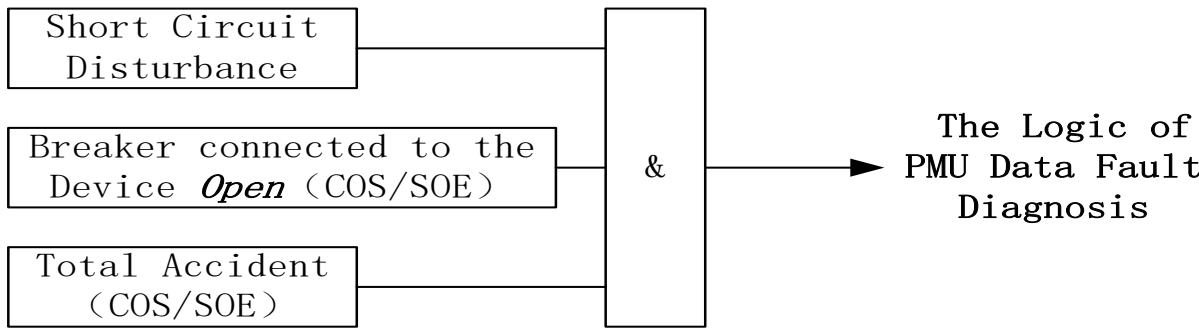

Figure 5. Fault diagnosis logic for PMU

1. PMU with two terminal configurations.

Judge Fault Phase: scan each phase, the phase is short circuit if satisfied Eq. 1.

$\mid$ Is $+\mathrm{Ie}|>0.5| \mathrm{Is}-\mathrm{Ie} \mid$

Is, Ie stands for two terminal current respectively.

If satisfied Eq. 2, then it is grounding short circuit, otherwise it is a phase to phase (two phase and three-phase ) short circuit.

$|\mathrm{Ia}+\mathrm{Ib}+\mathrm{Ic}|=|3 \mathrm{I} 0|>\mathrm{Iset}$

Iset is a larger current value,

Reclosed action situation, when short circuit occurs, use Eq. 3 per $10 \mathrm{~ms}$ for each phase of the fault line

$\mid$ Is $+\mathrm{Ie}|>0.5|$ Is-Ie $\mid$

Use Eq. 4 to check whether there is a fault current

$\mid$ Is $\mid<$ Izero

Izero stands the maximum charge current after the line opened.

2. PMU with signal terminal configuration.

Get the line reactance XL or ZL, Calculate the fault loop distance impedance $|\mathrm{Zm}|$, compared with 0.8 times the positive impedance of the line $\mathrm{Z} 1$; if $|\mathrm{Zm}|<0.8 \mathrm{Z} 1$, then short circuit.

\section{Fault Diagnosis base on Fault Recorder.}

After receiving the fault report, whether there is breaker trip signal within 5 minutes, if it does meet the condition above, send fault alarm.

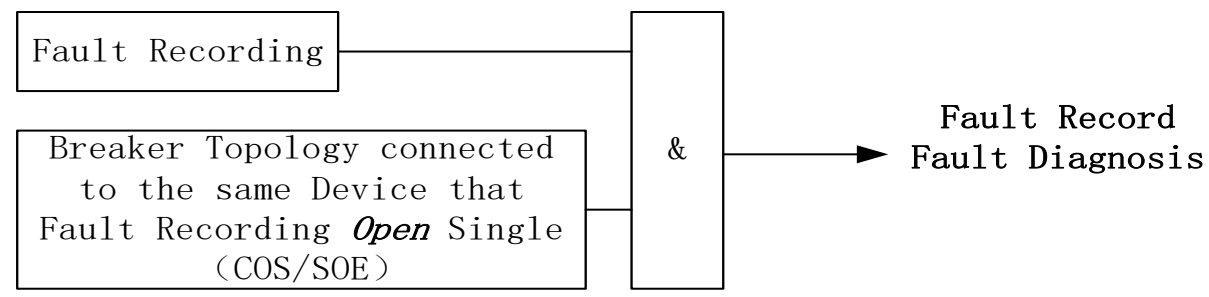

Figure 6. Fault diagnosis logic for Fault Record

\section{Assistant Decision Making Based on Fault Diagnosis Logic}

Establish the fault event list index by failure time and equipment, According to the priority of different alarm information show in Table 2 (the bigger value the higher priority), the analysis results of different sources of the same fault will be integrated, improve the fault briefing, and complete the report to guide the fault disposal [10].

Power Grid Intelligent Diagnosis and Decision Module.

When the fault occurs, determine the fault equipment, phase, nature and whether the trip equipment has the power supply conditions. 


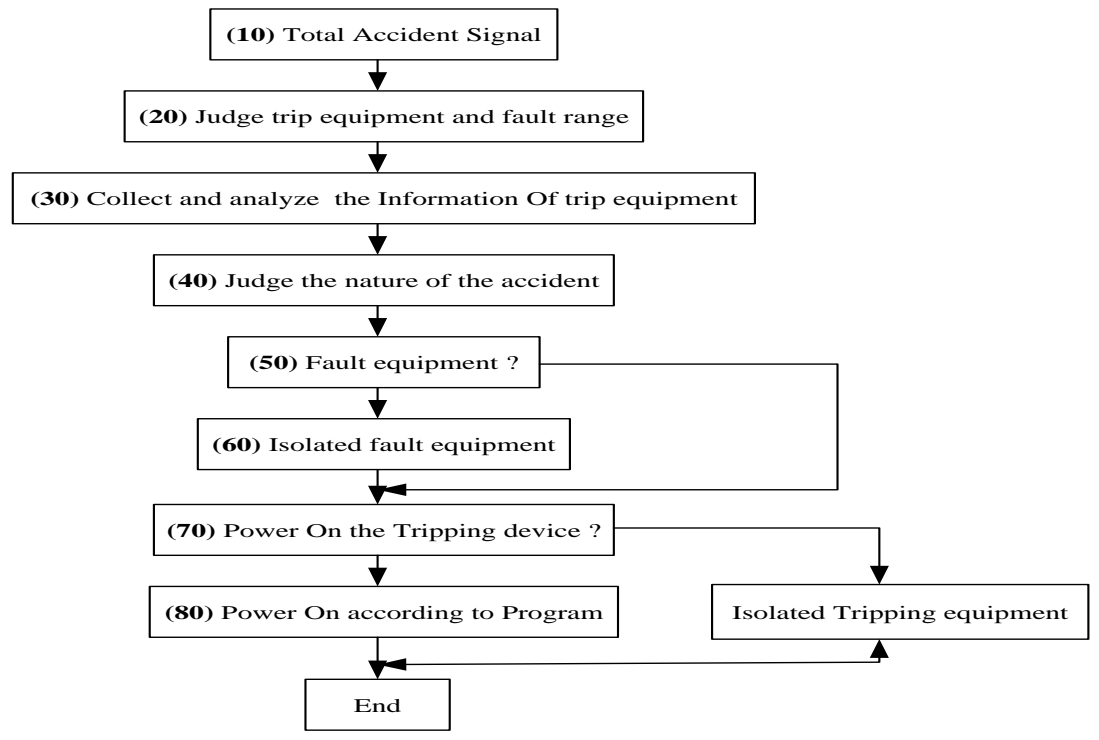

Figure 7. Power grid fault intelligent diagnosis process

Step 50, Judge damage equipment according to steady state information, When the locking signals such as SF6 low pressure locking, breaker failure protection action, etc. Make the judgment that the equipment is damage and need to be isolated. The difference between step 50 and 70,80 that step 50 in case of multiple components trip, the damaged components need to be isolated, the other components has the power supply conditions. Step 70 and 80 only a single components trip and is not able to power supply. The situation that can't be forced to power supply shown in table 3 .

\section{Device Abnormal Diagnosis Module.}

Given processing scheme according to the equipment abnormal diagnosis conclusion, through the picture integration display.

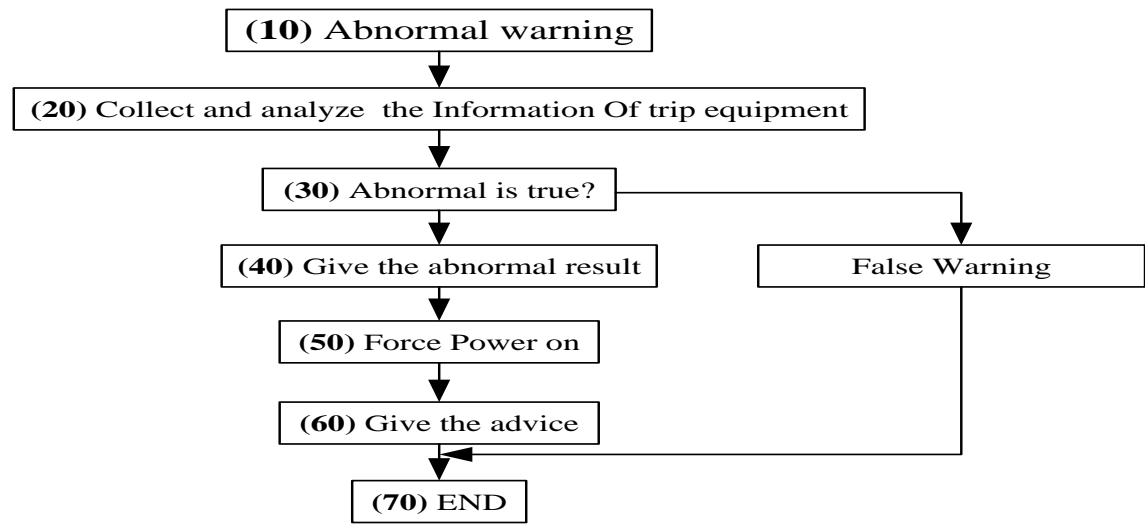

Figure 8. Device abnormal diagnosis process

\section{Application Case}

Intelligent analysis and fault diagnosis method based on multi-source information is a core function of intelligent power grid Automation system. It is constructed and demonstrated applications in $500 \mathrm{kV}$ Nanning area control center, Extra High Voltage Transmission Company, China Southern Power Grid. It will demonstrate two examples below: 500kV Power transmission Line Single phase reclosed failed and 500kV Breaker low oil pressure locking.

Example 1: 500kV Power Transmission Line Single Phase Reclosed Failed. 

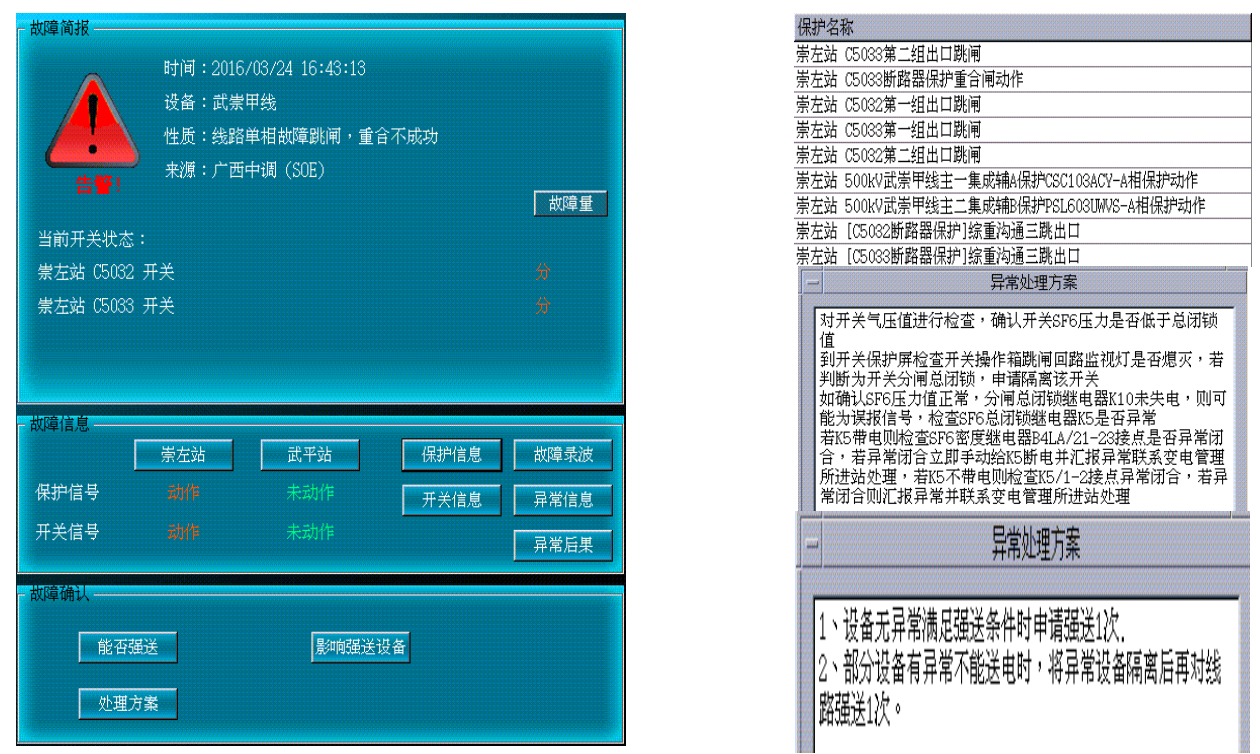

Figure 9. 500kV Power transmission Single phase reclosed failed

\section{Conclusions}

This paper introduced intelligent power analysis and fault diagnosis method based on multi-source information, it realize the information sharing and Providing technical support for the collaborative processing of warn information. By relating knowledge, give the auxiliary decision. It improves the accuracy of fault handling effect and ensures the operation of power grid safety. It is valuable to be popularized.

\section{Example 2: 500kv Breaker Low Oil Pressure Locking.}

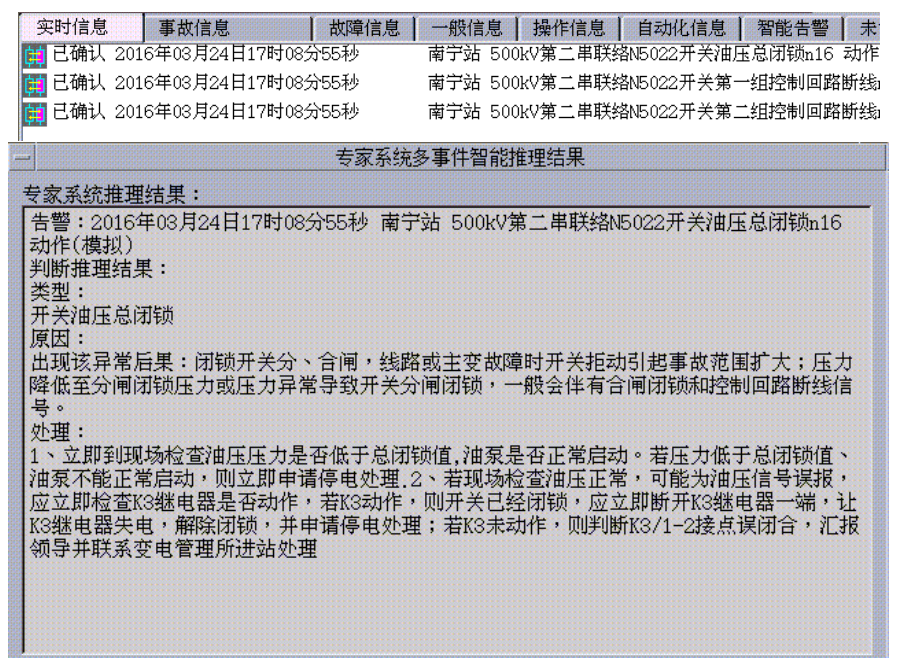

Figure 10. 500kV Breaker low oil pressure locking

\section{References}

[1] Haitao Luo. DEVELOPMENT OF DISPATCHINGAUTOMATION TECHNOLOGY IN CHINA -FROM SCADA TO EMS, Power System Technology, 2004, 28(4):43-46.

[2] GUO Chuang-xin, YOU Jian-xun, PENG Ming-wei, et al. A fault intelligent diagnosis approach based on element-oriented artificial neural networks and fuzzy integral fusion. Transactions of China Electrotechnical Society, 2010, 29(9): 183-190.

[3] YANG Wei-jian, HE Zheng-you, ZANG Tian-lei. Power system fault diagnosis method based on directional weighted fuzzy Petri nets. Proceedings of the CSEE, 2010, 30(34): 42-49. 
[4] ZHAO Wei, BAI Xiao-min, DING Jian, etc al. A new fault diagnosis approach of power grid based on cooperative expert system and multi-agent technology. Proceedings of the CSEE, 2006, 26(20): $1-8$

[5] ZhangJing, Cheng Xilai, Research on the application of multi-source heterogeneous data integration [J], Journal of chengdu university of information technology, 2009, 24(3).

[6] HUANGLin,WUZhi-jie,HUANGXiao-fang,WEIYong,FUZhi-hui. An improved multi source heterogeneous alert aggregation scheme $[\mathrm{J}]$. Computer application research, 2014, 31(2):579-582.

[7] GUO Chuang-xin, PENG Ming-wei, LIU Yi. Novel approach for fault diagnosis of the power grid with information fusion for multi-data resource. Proceedings of the CSEE, 2009, 29(31): $1-7$.

[8] GUO Chuang-xin, GAO Zhen-xing, LIU Yi, et al. Hierarchical fault diagnosis approach for power grid with information fusion using multi-data resources. High Voltage Engineering, 2010, 36(12): 2976-2983.

[9] Deng Guoxin, Research on fault information identification in regional power grid fault diagnosis system,North China Electric Power University (Beijing) 2008.

[10] Yang Honggeng, Ming Jiao, Dai Haibo, Implementation of intelligent alarm system for regional power grid, Electric power system and its automation2011, 23(2):105-109. 